\title{
Numerical modelling of gas-solid interface for homogeneous propellant combustion
}

\author{
A. Peratta \\ Wessex Institute of Technology, Southampton, UK
}

\begin{abstract}
This paper reports on the implementation and results obtained with a timedependent Arbitrary Lagrangian-Eulerian Finite Volume calculation for modelling the fluid-solid interface in laminar burning of solid energetic materials. The formulation is based on the conservation equations of mass, momentum and energy troughout the moving interface. Degradation-pirolysis and combustion are taken into account in the solid and gase phase, respectively. The chemical model is implemented with 7 unidirectional global reactions and 12 reactive species. The outcomes of the model are pressure, temperature, density, heat release, species concentration, gas speed and bulk burning rate. The numerical model is able to describe the main characteristics of the flame structure, including the induction (dark) zone. Burning rates and species concentration profiles are in good agreement with experimental measurements and previously published literature. The method can be used to study a variety of time-dependent processes including transient ignition, extinction, and combustion instabilities.
\end{abstract}

\section{Introduction}

Solid energetic materials are mostly used for rocket propulsion systems in either space or defence industry. Other applications include pyrotechnic actuators, boosters, manoeuvring and safety devices, as well as air-bags for automobile safety [1] and gas generators. Among the large family of solid energetic materials are the "smokeless" homogeneous propellants, developed several decades ago, which are still in use in many of the above mentioned applications. The homogeneous propellant, also known as Double-Base propellant (DBP) carries both oxidising and reducing chemical agents in the same molecular structure, usually composed of a homogeneous mixture of Nitroglycerine (NG) and 
Nitrocellulose (NC) with some plasticiser and other minor ingredients [2, 3]. ( $\mathrm{NG}=\mathrm{C}_{3} \mathrm{H}_{5} \mathrm{O}_{3}(\mathrm{NO} 2)_{3}$, and $\mathrm{NC}=\mathrm{C}_{6} \mathrm{H}_{7} \mathrm{O}_{2}\left(\mathrm{NO}_{3}\right)_{3}$, respectively.) Because of this feature, the combustion of DBPs can be regarded as preliminary mixed from the beginning of the process. Although DBP don't usually have high specific impulses $\left(I_{s} \lesssim 210 s\right.$ under normal conditions), they are still on high demand due to its smokeless combustion, and their relatively easy manufacturing, they can be made by solvent or semi-solvent processes and extruded in various shapes. Hence, there is no need to keep into account mixing processes between oxidiser and reducer. Much effort has been done in research oriented to the CFD applied to solid propellant combustion, see for example $[4,5]$.

The aim of this work is to develop a comprehensive numerical modelling for the unsteady chemically reactive compressible multi-species flow interacting with the solid reactive burning surface of the DBP.

The model keeps track of the moving boundary as the solid burns, thus allowing to obtain a bottom-up prediction of the burning rate. The paper is organised as follows, in Section 2 the theoretical formulation is established. Section 3 provides a brief description of the DB under study. Section 4 describes the interface boundary conditions, Section 5 presents the numerical scheme. Section 6 shows the numerical results obtained in a one-dimensional implementation and finally Section 7 elaborates the corresponding conclusions.

\section{Theoretical formulation}

The theoretical formulation [6] is based on the following average conservation of mass, species concentration, momentum and energy equations for a mixture of $N_{e}$ species and $N_{r}$ global chemical reactions:

$$
\begin{array}{rlrl} 
& \frac{\partial \rho}{\partial t}+\nabla \cdot(\rho \mathrm{u}) & =0 \\
& \text { Mass } & \frac{\partial\left(\rho Y_{k}\right)}{\partial t}+\nabla \cdot\left(\rho \mathrm{u} Y_{k}+\mathrm{q}_{k}^{D}\right) & =\omega_{k} \\
& \text { Species } & \frac{\partial(\rho \mathrm{u})}{\partial t}+\nabla\left(\mathrm{u}^{t} \rho \mathrm{u}+p+\bar{\sigma}\right) & =0 \\
\text { Momentum } & \frac{\partial(\rho \varepsilon)}{\partial t}+\nabla \cdot\left(\rho \mathrm{u} \varepsilon+\mathrm{q}^{e}\right)+p \nabla \cdot \mathrm{u} & =-\sum_{k=1}^{N_{e}} \omega_{k} \Delta h_{k}^{T_{r}},
\end{array}
$$

where subscript $k$ denotes species, $\omega_{k}$ is the reaction constant, $Y_{k}$ the mass fraction, $p$ pressure, $\mathrm{u}, \rho$ and $\varepsilon$ are the average velocity, density and internal energy, respectively, defined as: $\rho \mathrm{u}:=\sum_{k=1}^{N_{e}} \rho_{k} \mathrm{u}_{k}$ and $\varepsilon:=\sum_{k=1}^{N_{e}} Y_{k}\left(\varepsilon_{k}-\varepsilon_{k}^{T_{r}}\right)$, where $\varepsilon_{k}=\varepsilon_{k}^{T_{r}}+\int_{T_{r}}^{T} c_{v, k}\left(T^{\prime}\right) d T^{\prime}$, being $T$ and $T_{r}$ the actual and reference temperatures, respectively. 


\subsection{Chemical model, thermodynamic and transport properties}

The specific rate of $j$-th global reaction $\kappa_{j, \beta}$ is calculated by mean of Arrhenius expressions of the form: $\kappa_{j}=B_{j} \exp \left[-E_{0} / R_{0} T\right]$, where $E_{0}$ is the activation energy, $R_{0}$ is the universal gas constant, and $B_{j} \approx$ cte $T^{\alpha}$ is the frequency factor with $0<\alpha<0.5$ and index $j$. Thus, $j$-th rate reaction can be calculated by means of: $\omega_{j}^{R}=\kappa_{j, f} \prod_{k=1}^{N_{e}} C_{k}^{\nu_{k j}^{*}}$, where $\nu_{j k}^{*}$ is the stoichiometric coefficient of the reactive species $k$ in reaction $j$, and $C_{k}$ is the mole concentration of the species.

The following equation of state (EOS) is considered $p=\rho R_{0} T \sum_{k=1}^{N_{e}} Y_{k} / M_{k}$ in the gas phase, where $c_{s}^{2}=p / \rho\left(1+R_{\text {mix }} / c_{v \text { mix }}\right) ; M_{k}$ is the molecular mass, $R_{\text {mix }}:=R_{0} \sum_{k=1}^{N_{e}} Y_{k} / M_{k}$ the mixture constant, $c_{v \text { mix }}=\sum_{k=1}^{N_{e}} Y_{k} c_{p k}-R_{\text {mix }}$ the mixture specific heat, and $c_{s}$ the speed of sound. The condensed phase (CP) is considered incompressible, i.e. infinite sound speed. The specific heat at constant pressure of each component is evaluated using:

$c_{p k}(T)=A_{k}+B_{k} T+C_{k} T^{2}+D_{k} T^{3}+E_{k} / T^{2}$, where $A, B, C, D$ and $E$ are constants obtained from [9-11]. Then, the specific internal energy of the mixture becomes: $c_{v, \text { mix }}=\sum_{k=1}^{N_{e}} Y_{k} c_{p k}-R_{\text {mix }}$.

Transport coefficients of species involved in energetic materials are not always available in published tables, therefore, they have to be approximated from the classical statistical theory of gases. The diffusive effects considered in eqs. (1) to (4) are viscosity, Fick's diffusion and Fourier law for heat conduction. In this way $\mathrm{q}_{k}^{D}=-\rho D_{k, \text { mix }} \nabla Y_{k}$ in (2) is the Fick's mass diffusive flux, $\sigma$ is the viscous tensor in (3), and $\mathrm{q}^{e}=-\lambda_{\operatorname{mix}} \nabla T-\rho \sum_{k=1}^{N_{e}} h_{k} Y_{k} u_{k}-\nabla(\bar{\sigma} \mathrm{u})$ is the diffusive flux of internal energy. The binary diffusion coefficient between species $\mathrm{A}$ and $\mathrm{B}$ is calculated by mean of the Chapman-Enskog $(\mathrm{C}-\mathrm{E})$ theory and the transport Boltzmann equation [9], the final results is:

$$
D_{A B}=\frac{3}{16} \frac{\left(\frac{8 \pi k_{B} T}{\mu_{A B}}\right)^{1 / 2}}{n \pi \sigma_{A B}^{2} \Omega_{D}} f_{D}
$$

where $n$ is the number density of molecules in the mixture, $\Omega_{D}$ the collision integral, $\sigma_{A B}$ the Lennard-Jones $(\mathrm{L}-\mathrm{J})$ mixture length scale, $f_{D}$ is the potential shape factor, $k_{B}$ is the Boltzmann constant, and $\mu_{A B}=m_{A} m_{B} /\left(m_{A}+m_{B}\right)$ is the reduced mass of the binary system. For the multi-component mixture, the effective diffusion coefficient $\left(D_{k, \text { mix }}\right)$ of $k$ with respect to the mixture is related to $D_{A B}$ by mean of: $D_{k \text {, mix }}=\left(1-X_{k}\right) / \sum_{j \neq k}^{N_{e}} \frac{X_{j}}{D_{k j}}$. Viscosity for a single component is calculated using $\mathrm{C}-\mathrm{E}$ theory and $\mathrm{L}-\mathrm{J}$ potential according to: $\eta=5 / 16 \sqrt{\pi m k_{B} T} /\left(\pi \sigma^{2} \Omega_{D}\right)$. Then, in order to obtain an average value for the mixture $\eta_{\text {mix }}$, Wilke's mixing rule is applied [9] yielding:

$$
\eta=\sum_{i=1}^{N_{e}} \frac{X_{i} \eta_{i}}{\sum_{j=1}^{N_{e}} X_{j} \varphi_{i j}} \quad \text { and } \quad \varphi_{i j}=\frac{\left[1+\sqrt{\eta_{i} / \eta_{j}}\left(M_{j} / M_{i}\right)^{1 / 4}\right]^{2}}{\sqrt{8\left(1+M_{i} / M_{j}\right)}} .
$$


C-E theory can also be applied to obtain the thermal conductivity $\lambda$ of pure mono-atomic species with no internal degrees of freedom [9, 10], yielding: $\lambda=25 / 32 \sqrt{\pi m k_{B} T} c_{v} /\left(\pi \sigma^{2} \Omega_{D}\right)$. Then, Eucken's correction factor given by: $\lambda M \eta^{-1}=1.32 c_{v}+1.77 R_{0}$, is used to consider the internal degrees of freedom in polyatomic molecules, and finally, for the multi-component mixture, Wilke's rule is used in the same way as for the viscosity coefficient $(\eta)$ above. Reference values for the calculation of cross sections, dipole moments, and collision integrals can be obtained from $[9,10,11]$.

\section{The DB propellant}

The extruded Ballistite, the Russian Cordite, or the composite or the cast DBP are the most common homogeneous compositions. In this work the EC1 formulation ( $52 \% \mathrm{NG}+43 \% \mathrm{NC}+5 \%$ of minor additives) contemplated by A. Kubota [2], with $1100 \mathrm{cal} / \mathrm{g}$ of heat of explosion has been adopted. Because of its relatively large stability under room conditions, an external source of heat (igniter) must be provided to initiate the DBP burning. During the ignition process, heat is transported by conduction inside the solid and the degradation reaction of $\mathrm{NG}$ and $\mathrm{NC}$ begins to accelerate generating reactive species like $\mathrm{NO}_{2}$ and aldehydes - see reaction (6). Then, further recombination takes place



Figure 1: Temperature, species and regions in condensed and gas phase near the burning surface of the DBP.

into the solid phase, as contemplated by reaction (7) yielding extra heat to reinforce the ignition. We call condensed phase (CP)to the region between gas and solid phase where solid propellant coexists with degradation and recombination products. (The CP consists of a pre-heated zone and a superficial degradation layer where molecular degradation of the propellant and further recombination of the decomposed products takes place.) As degradation evolves, the solid concentration drops down from one to zero. The closer to the burning surface the higher degradation rates. When solid concentration reaches zero at the interface, convection process drags the unburnt condensed phase from the cold zone of the DBP thus replacing the decomposed material. Then, a convective chemically reacting flow emerges from the burning surface toward the gas phase with a 
velocity ruled by eq.(1). The emerging molecules react with each other in the gas phase, developing an important amount of heat in the flame zone. The distribution of heat release along the flame zone is ruled by the coupling between the gas-phase reactions, hydrodynamics, and transport phenomena, thus yielding a particular flame structure as qualitatively shown in Figure 1. Meanwhile, the heat released in the primary zone provides some feedback energy to maintain the degradation reaction in the CP. The combustion can be either self-sustained steady, unsteady, oscillatory, or vanishing, depending on this energetic loop.

The modelling involves $N_{r}=7$ chemical reactions. The following two global ones, representing DB decomposition and endothermic recombination respectively, take place in the $\mathrm{CP}$ :

$$
\begin{aligned}
& \mathrm{DB} \rightarrow 2.49 \mathrm{NO}_{2}+2.36 \mathrm{CH}_{2} \mathrm{O}+1.26(\mathrm{CHO})_{2}+0.17 \mathrm{CO}+\mathrm{mr} \\
& \mathrm{NO}_{2}+0.56 \mathrm{CH}_{2} \mathrm{O}+0.16(\mathrm{CHO})_{2} \rightarrow \mathrm{NO}+0.38 \mathrm{CO} \\
&+0.5 \mathrm{CO}_{2}+0.5 \mathrm{H}_{2} \mathrm{O}+0.22 \mathrm{H}_{2} \\
& \mathrm{NO}_{2}+0.56 \mathrm{CH}_{2} \mathrm{O}+0.16(\mathrm{CHO}) 2 \rightarrow \mathrm{NO}+0.38 \mathrm{CO}+0.5 \mathrm{CO}_{2} .
\end{aligned}
$$

The 5 remaining reactions taking place in the gas phase are given in ref. [11, 12]. The simulation involves the following species: $\mathrm{DB}, \mathrm{NO}_{2}, \mathrm{CH}_{2} \mathrm{O},(\mathrm{CHO})_{2}, \mathrm{CO}$, $\mathrm{NO}, \mathrm{CO}_{2}, \mathrm{H}_{2} \mathrm{O}, \mathrm{H}_{2}, \mathrm{C}_{2} \mathrm{H}_{4}, \mathrm{~N}_{2}$ and $\mathrm{C}$. The corresponding rate reactions, specific enthalpy, and Arrhenius coefficients were obtained from [11, 13, 14].

\section{Interface boundary conditions}

Gas and CP equations couple to each other throughout the burning surface (interface) by means of the following mass and species conservation equations:

$$
[\rho u]_{\mathrm{gas}}=\rho_{p} r_{b}, \quad \text { and } \quad\left[\rho \mathrm{u}_{k} \cdot \hat{n} Y_{k}\right]_{\mathrm{gas}}=\rho_{p} r_{b} Y_{k},
$$

respectively, and energy according to:

$$
\left[-\lambda_{\operatorname{mix}} \frac{\partial T}{\partial \hat{n}}+\rho \sum_{k=1}^{N_{e}} Y_{k} h_{k} \mathrm{u}_{k} \cdot \hat{n}\right]_{\mathrm{gas}}=-\lambda_{s} \frac{\partial T}{\partial \hat{n}}+\rho r_{b}\left[c_{p}\left(T_{s}-T_{r}\right)+\sum_{k=1}^{N_{e}} Y_{k} \Delta h_{k}\right]_{s}
$$

where subscript $s$ refers to the $\mathrm{CP}, r_{b}$ is the local propellant burning rate, $T_{s}$ is the interface temperature, $h$ is enthalpy and $\Delta h$ the enthalpy at reference temperature. Because reactions in the $\mathrm{CP}$ have relatively high activation energy and occur in a very thin region (few microns in foamy zone), a matched asymptotic expansion technique as described in [13-15] can be used in order to estimate the burning rate formula. The result for a steady self-sustained combustion is:

$$
r_{b}^{2} \approx \beta^{\prime} \frac{T_{s}^{2}}{T_{s}-T_{0}-Q_{s} /\left(2 c_{s}\right)} \exp \left[\frac{-E_{c}}{R_{0} T_{s}}\right]
$$

where $\beta^{\prime}:=\lambda_{s} B c R_{0} /\left(c_{S} \rho_{s} E_{c}\right)$ is a constant, $T_{0} \approx 293 K$ is the initial temperature equal to the temperature at $x \rightarrow \infty$ in the cold zone of the propellant. 
The following values were assumed: $\rho_{s}=1.6 \mathrm{~g} / \mathrm{cm}^{3}, c_{s}=0.4 \mathrm{cal} /(\mathrm{gK}), \lambda_{s}=$ $5.1 \times 10^{-2} \mathrm{cal} /(\mathrm{smK}), B_{c}=10^{17} \mathrm{~s}^{-1}$, and $E_{c}=40 \mathrm{kcal} / \mathrm{mol}$. Thus $r_{b} \approx$ $10^{-2} \mathrm{~m} / \mathrm{s}$, and consequently: $\delta x_{c} \approx 37 \mu \mathrm{m}, \tau_{c} \approx 4 m s, \delta x_{d} \approx 2 \mu m, \tau_{d} \approx 0.2 \mathrm{~ms}$, where $\delta x$ indicates characteristic length, $\tau$ residence time, and subscripts $c$ and $d$ pre-heated and foamy zone in the $\mathrm{CP}$, respectively. Note that the typical Mach number in the flame zone is $M \approx 10^{-3}$, this involves an incompressible flow regime which might potentially limit the numerical scheme.

\section{Numerical scheme}

An Implicit Continuous Eulerian (ICE) method [16] with Arbitrary LagrangianEulerian (ALE) technique was implemented in order to solve the 1D flame structure near the interface of the DBP. The scheme is based on the Finite Volume Method (FVM) in which each physical process is integrated in an independent way during the calculation time step. The scheme uses a Staggered Grid approach where all diffusive fluxes (q) and flow velocities (u) are assigned to vertices (representing cell faces) whereas $\varepsilon_{\text {mix }}, T, p$, cell volume $V$, and $Y_{k}$ are assigned to cell centres as shown in Figure 2(a). Any time variation of a cell dynamic properties $\psi$ is calculated as $\delta \psi=\psi^{m+1}-\psi^{m}$, where $m$ indicates time level, Time variations due to either Fick's mass diffusion, Fourier thermal diffusion, or energy diffusion due to species migration is calculated in a Lagrangian way with a fully implicit time integration method of the form: $\delta Y_{k}=Y_{k}^{m+1}-Y_{k}^{m} \approx \delta t \frac{d}{d t} Y_{k}^{m+1}$, in case of mass diffusion, where $d Y_{k} / d t$ at the advanced time level can be obtained by means of:

$$
\frac{d\left(m_{i} Y_{i}^{k}\right)}{d t} \approx \gamma_{i-\frac{1}{2}}^{k}-\gamma_{i+\frac{1}{2}}^{k} \text {, and } \gamma_{i-\frac{1}{2}}^{k}=\left(\rho_{k} D_{k, \text { mix }}\right)_{i-\frac{1}{2}}\left(\frac{Y_{i-1}^{k}-Y_{i}^{k}}{V_{i}+V_{i-1}}\right) A .
$$

$A$ represents cross section, $V$ the cell volume, and $m$ the total mass within the cell. Next, hydrodynamic terms are integrated with a modified ICE method (Implicit Continuous Eulerian) $[11,16]$. Source terms in (2) and (4) from chemical reactions are then explicitly integrated in time, according to:

$$
\delta Y_{k, i}^{m+1}=\frac{\omega_{k, i}^{m}}{\rho_{i}^{m}} \delta t, \quad \text { and } \quad \delta(m \varepsilon)_{i}^{m+1}=V_{i}^{m} \sum_{k=1}^{N_{e}} \omega_{k, i}^{m} \Delta h_{k} \delta t
$$

Each integration is performed in a Lagrangian way, thus cancelling all convective terms. Then, after each calculation cycle, vertices are moved arbitrary over the fluid in order to dynamically adapt the mesh to the solution. As a consequence, convective terms are artificially restored. This grid reorganisation allows to automatically tune the grid resolution at each time level according to the gradients of $Y_{k}, T$ and heat release. In this work, the integral of the following weight function in space has been equi-distributed in each cell by adjusting vertices location:

$$
w=c\left(1+b_{1} \frac{\partial T}{\partial x}+b_{2} \frac{\partial \omega_{k} \Delta h_{k}}{\partial x}\right),
$$


where $b_{1}$ and $b_{2}$ are constants and $c$ is determined such that $w(x)$ is normalised along the gas phase, i.e.: $\int_{0}^{L} w\left(x^{\prime}\right) d x^{\prime}=1$. A detailed explanation of the technicalities of the scheme and the pseudo-code is presented in $[11,12]$.
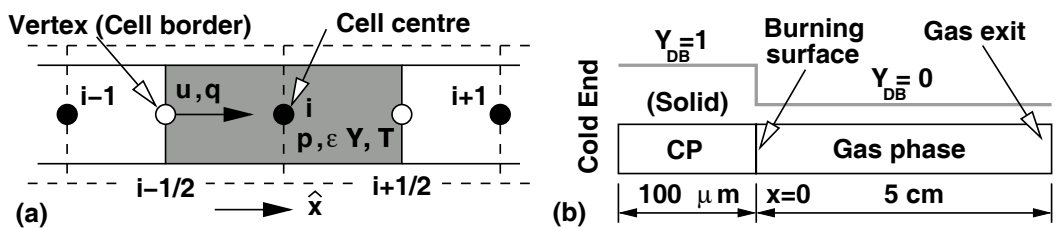

Figure 2: (a) Staggered grid for the DBP flame structure modelling; (b) 1D conceptual model, showing distribution of $Y_{\mathrm{DB}}$ and the slab dimensions at initial time $(t=0)$.

\section{Results}

The ICE-ALE FV method has been applied to the 1D combustion of the DBP shown in Figure 2(b). The burning surface is located at $x=0$ where the FV version of matching conditions (8),(9) are prescribed. The cold end of the DBP has $T=293 K, Y_{\mathrm{DB}}=1$, and $Y_{k}=0$ for $k \neq D B$. Boundary conditions are implemented by mean of phantom cells and vertices. Smooth outflow boundary conditions like $\partial\left(u_{x}, \varepsilon, Y_{k}\right) / \delta x=0$ are prescribed at the gas exit. The initial conditions are $Y_{\mathrm{DB}}=1, Y_{\mathrm{N} 2}=0, T=293 K$ at $x \leq 0$, and $Y_{\mathrm{DB}}=0, Y_{\mathrm{N} 2}=1$, $T=650 K$ at $x>0$. An external source of heat has been added in order to maintain a temperature of $650 \mathrm{~K}$ during $1 \mathrm{~ms}$ so that reactions (6) and (7) can start. Once the combustion begins, this transient condition is removed, and later on a self-sustained laminar combustion is achieved. All transient effect due to ignition and flame build-up vanish after approximately $t=0.2 s$ when the process becomes stable. Figure 3 shows the concentration profile of all species in gas phase and $\mathrm{CP}$ at $t=0.2 \mathrm{~s}$.

Figures 4 and 5 show the temperature profiles before and after the formation of the induction zone [15], near the interface. The results obtained for temperature and species concentration are in excellent agreement with the ones previously obtained in [14] and others qualitative descriptions of the flame structure [15]. Figure 6 shows a comparison between the Saint-Robert's burning law (Vieille's law) where $R_{b}=A p^{n}$, and the results of the present simulation. A mean squares fit of the results gave an exponent $n=0.765$, which is typical of the DBP of the kind used in this investigation. 

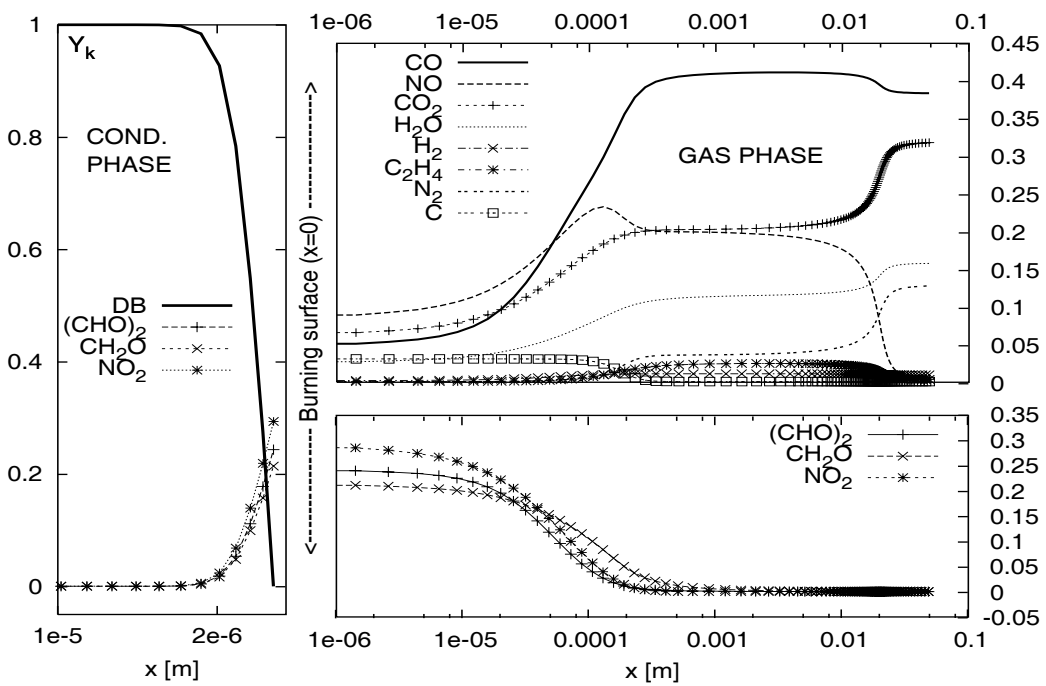

Figure 3: Species concentration profile near the burning surface in gas and condensed phases at $t=0.2 \mathrm{~s}$ when the combustion process has achieved a steady self-sustained state.
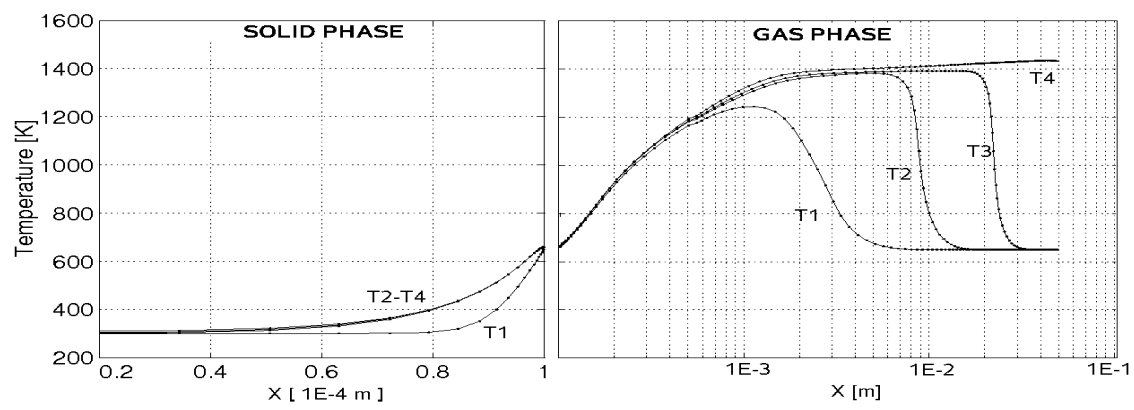

Figure 4: Temperature profiles in the solid and gas phase at times $T_{1}=0.0024 \mathrm{~s}$, $T_{2}=0.0036 \mathrm{~s}, T_{3}=0.0062 \mathrm{~s}$, and $T_{4}=0.0140 \mathrm{~s}$ after the ignition. Note that the solid phase has been represented in linear scale while the gas phase in logarithmic scale.

\section{Conclusions}

The hybrid FVM - ALE scheme results adequate for accurate quantitative studies of time-dependent combustion properties of solid energetic materials with moving boundaries in laminar regime. The present approach is in principle oriented to compressible flow, however, it resulted quite satisfactory for modelling low Mach numbers, i.e. $O\left(10^{-3}\right)$. The burning rate is obtained as a natural consequence of 


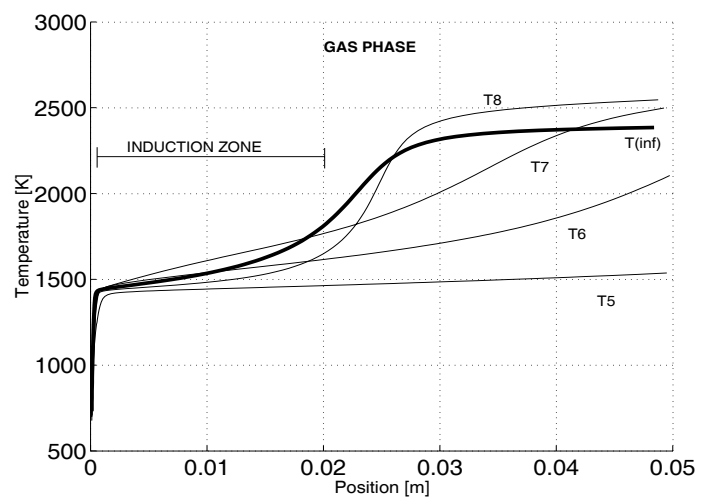

Figure 5: Temperature profile during the formation period of the induction zone at times $T_{5}=0.0480 \mathrm{~s}, T_{6}=0.0623 \mathrm{~s}, T_{7}=0.0633 \mathrm{~s}, T_{8}=0.0675 \mathrm{~s}$, and $T_{\text {inf }}>0.2 \mathrm{~s}$

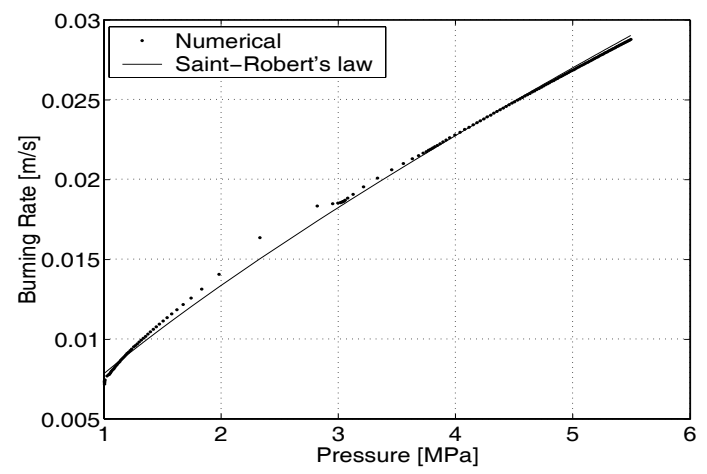

Figure 6: Burning rate vs. pressure. Comparison between numerical finding and the well established Saint-Robert's law.

the gasification of the last cell in the condensed phase. The comparison between Saint-Robert's burning law and numerical findings is very good once the flame achieved a steady state. The scheme has proven to be stable and provided accurate description of the flame structure and flame dynamics near the burning surface of the propellant. Hence, it can be used to study a variety of time-dependent combustion processes including transient ignition and combustion instabilities.

\section{References}

[1] P. P. Cord and F. Beteille. Versatility of a two chamber inflator for airbag application. In Airbag 2004, 7th International Symposium and exhibition on Sophisticated car Occupant Safety Systems, Karlsruhe, Allemagne, 2004. 
[2] Aoki N. Kubota. Combustion wave structures of high- and low- energy double-base propellants. AIAA Journal, 20(1):100-105, 1982.

[3] E. Dick, J. Bulgheroni, R. Crespo, J. Padula, E. Runstein, and L. Urdiain. La propulsion de cohetes y misiles (in spanish). Technical Report 685, CITEFA, Bs As, Argentina, 1989.

[4] M. T. Health, R. A. Fiedler, and W. A. Dick. Simulating solid propellant rockets at csar. AIAA Paper, pages 2000-3455, 2000.

[5] H. J. Gibeling, R. K. Madabhushi, F. J. De Long, and J. S. Sabnis. Advanced flow field model. Technical report, U.S.Air Force Philips Lab., 1993. CRPLTR-3005, Edwards AFB CA.

[6] J. Warnatz, U. Maas, and R.W. Dibble. Combustion. Springer Verlag, Berlin, 1996.

[7] D.R. Stull and H. Prophet. Janaf tables of thermochemical properties, 2nd ed. Technical report, National Standard Reference Data System, Washington, 1971.

[8] National Institute of Standards and Technology. The NIST Chemistry WebBook. http://webbook.nist.gov/, 2005. U.S. Secretary of Commerce.

[9] Hirschfelder, Curtis, and Bird. Molecular Theory of gases and Liquids. John Wiley \& Sons, Inc., New York, 1954.

[10] B. E. Poling, J. M. Prausnitz, and John P. O' Connel. The Properties of gases and Liquids. McGraw Hill, 5 edition, 2000.

[11] A. Peratta. CFD Aplicado a la Combustión de Propulsantes Sólidos Homogéneos. Master's thesis, University of Buenos Aires, Faculty of Exact and Natural Sciences, October 2001. in spanish.

[12] A. Peratta, C. González, and E. Dick. Numerical analysis for double-base propellant combustion. In V. Sanchez-Galvez and C.A. Brebbia, editors, Computational Ballistics II, volume 40 of Transactions on Modelling and Simulations, pages 269-278. Wessex Institute of Technology, WIT-Press, 2005.

[13] V. Yang, Thomas Brill, and Wu-Zhen Ren, editors. Solid Propellant Chemistry, Combustion, and Motor interior Ballistics, volume 185. American Institute of Aeronautics and Astronautics, New York, 2000.

[14] Tae-Seong Roh, I-Shih Tseng, and Vigor Yang. Effects of acoustic oscillations on flame dynamics of homogeneous propellants in rocket motors. Journal of Propulsion and Power, 11(4), July-August 1995.

[15] G. Lengellé, A. Bizot, J. Duterque, and J.F. Trubert. Fundamentals of solid propellant combustion. In K.K.Kuo and M. Summerfield, editors, Progress in Astronautics and Aeronautics, AIAA, volume 90, page 361, New York, 1984.

[16] C.W. Hirt, A.A. Amsdem, and J.L.Cook. An arbitrary lagrangian-eulerian method for all flow speeds. Journal of Computational Physics, 14:227-253, 1974. 